\title{
Crossing a narrow-in-altitude turbulent auroral acceleration region
}

\author{
R. Pottelette ${ }^{1}$, R. A. Treumann ${ }^{2,3, *}$, and E. Georgescu ${ }^{3}$ \\ ${ }^{1}$ Centre d'Étude des Environnements Terrestre et Planétaires, CNRS, 4 av. de Neptune, F-94107 St. Maur des Fossés, France \\ ${ }^{2}$ Centre for Interdisciplinary Plasma Science, Max-Planck-Institute for extraterrestrial Physics, P.O.Box 1312, D-85741 \\ Garching, Germany \\ ${ }^{3}$ Max-Planck-Institute for extraterrestrial Physics, P.O.Box 1312, D-85741 Garching, Germany \\ *Also at Department of Physics and Astronomy, Dartmouth College, Hanover, NH 03755, USA
}

Received: 21 October 2003 - Revised: 16 December 2003 - Accepted: 17 December 2003 - Published: 14 April 2004

Part of Special Issue "International Workshops on Nonlinear Waves and Chaos in Space Plasmas"

\begin{abstract}
We report on the in situ identification of a narrow electrostatic acceleration layer (electrostatic shock) containing intense plasma turbulence in the upward current region, and its effect on auroral particles. Wave turbulence recorded in the center of the layer differs in character from that recorded above and beneath. It is concluded that the shock is sustained by different nonlinear waves which, at each level, act on the particles in such a way to produce a net upward directed electric field. The main power is in the ion acoustic range. We point out that anomalous resistivities are incapable of locally generating the observed parallel potential drop.
\end{abstract}

\section{Introduction}

The origin of bright aurorae is understood to lie in the presence of mildly energetic electrons in the $\mathrm{keV}$ range that are accelerated towards the ionosphere by upward pointing electric fields (Block and Fälthammar, 1990; Boström et al., 1988). In contrast, black aurorae are attributed to the upward acceleration of ionospheric electrons by downward electric fields (Marklund et al., 1997; Marklund et al., 2001). In the recent past, measurements in situ the upward (Mozer and Hull, 2001; Boström, 1992a, b) and downward (Carlson et al., 1998b; Ergun et al., 1998; McFaden et al., 2003) auroral current regions of the Earth's auroral magnetosphere provided ample evidence for this kind of acceleration. The corresponding upward auroral electric fields in the upward current region accelerate the ionospheric ion component out into the magnetosphere to become an upward narrow-angle fieldaligned ion beam. At the same time the auroral electrons assume a more ring like velocity distribution as predicted by simple adiabatic models (Chiu and Schulz, 1978) of particle motion in the converging magnetic mirror geometry of the Earth's magnetic field and the electric potentials. Models of

Correspondence to: R. Pottelette

(raymond.pottelette@cetp.ipsl.fr) the acceleration potential region range from large-scale double layers to turbulent microscopic electric wave structures. Magnetic field-aligned accelerating electric potentials in the upward current region have also been inferred from the interpretation of remotely detected auroral kilometric radiation (AKR) fine structure events (Gurnett and Anderson, 1981; Pottelette et al., 2001). The FAST spacecraft, with its high time resolution instrumentation - regarding both wave and particle distributions - and high telemetry rate (Carlson et al., 1998a; Pfaff et al., 2001), has provided new details concerning the plasma processes occurring in the auroral acceleration region.

\section{Observations}

A textbook example of the particle and field signatures during a FAST spacecraft crossing of the up- and downward ionospheric current system is given in Fig. 1. These data are derived from one particular orbit which has been extensively studied previously (Elphic et al., 1998; Chaston et al., 2002). The dominant feature is seen in panel (e), where two brief low energy $(<$ few $\mathrm{keV}$ ) upward electron flux bursts at second 3 and second 68 frame a broad energetic $(\sim \mathrm{keV})$ downward "inverted- $V$ " electron event (traditionally called so because of its $\Lambda$-like signature in the electron energy spectra) which lasts for $55 \mathrm{~s}$ from second 8 to second 63 . The electron angle of flow with respect to the magnetic field is shown in panel (f) with $180^{\circ}$ for upward and $0^{\circ}\left(360^{\circ}\right)$ for downward directions.

At $8 \mathrm{~s}$, the entire ionospheric ion population (panel $(\mathrm{g})$ ) is accelerated upward $\left(180^{\circ}\right.$ in panel (h)) into an intense $(\sim \mathrm{keV})$ ion beam with practically no low energy ions left in the vicinity of the spacecraft. Panel (a) shows the magnetic signature corresponding to the field aligned currents carried by the particles. Downward currents have positive gradients in the magnetic field while the "inverted-V" upward current causes a negative gradient. 


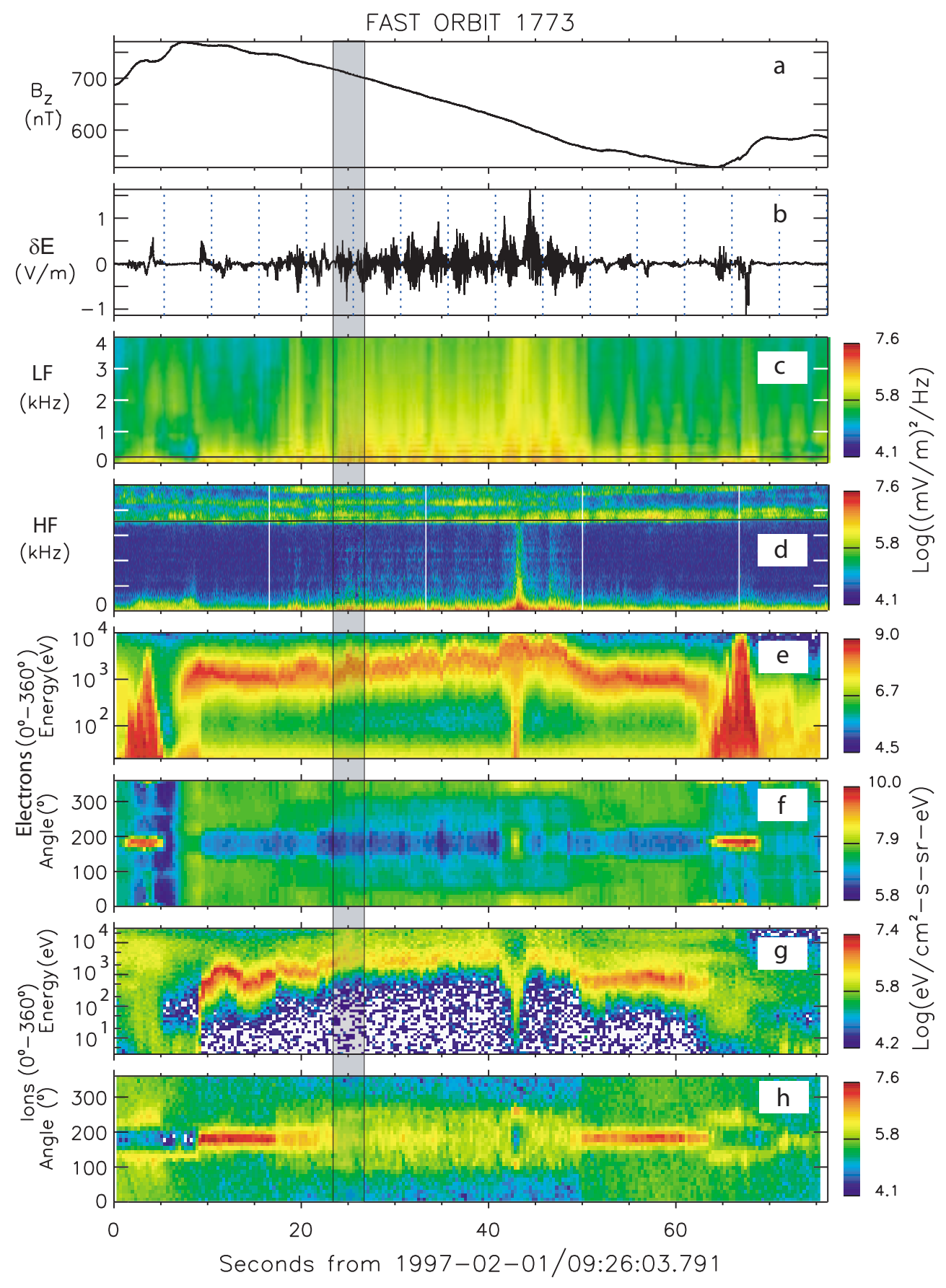

Fig. 1. Plasma measurements during a FAST spacecraft passage through an active auroral current system. The shaded stripe is analyzed in detail in the present Letter. (a): East-West magnetic field component resulting from the magnetic field-aligned currents carried by electrons and flowing in the auroral region during auroral activity. Positive slopes are caused by currents flowing from the magnetosphere downward into the ionosphere, negative slopes are signatures of upward currents. (b): Electric wave field. Note the high activity in the upward current region. (c): Low frequency spectrum of electric waves. The black line at $\sim 200 \mathrm{~Hz}$ is the ion cyclotron frequency $f_{c i}$. Broadband noise and ion cyclotron harmonics appear in the upward current region. (d): High frequency electric spectrum. The black line at $\sim 350 \mathrm{kHz}$ is the electron cyclotron frequency $f_{c e}$. At higher frequencies 3 auroral kilometric radiation bands appear. Higher frequency implies lower altitude above ground. Emission below $f_{c e}$ indicates closeness to the radiation source and acceleration region. (e): Electron energy-flux spectrogram indicating two downward current regions to the left and right and the broad upward current region. (f): The angular distribution of the electron energy flux of panel (e). Downward currents are indicated as upward flux $\left(180^{\circ}\right)$, upward current as downward flux $\left(0^{\circ}\right)$. $(\mathrm{g})$ : Same for ions. In the upward current region no low-energy ions exist. The ion spectrogram shows a nearly stationary band at a few keV energy. (h): Ion angular distribution. In the upward current region the ion fluxes are exclusively upward at $180^{\circ}$. 


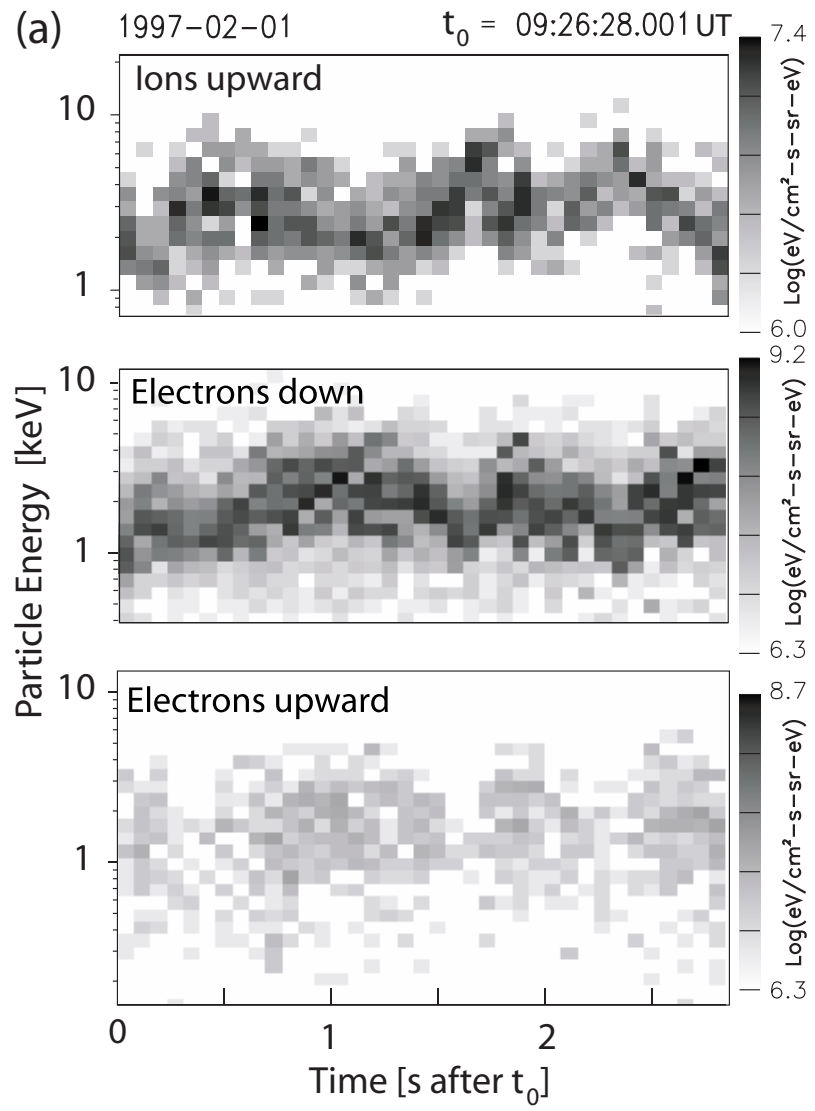

(b) Electron Temperature
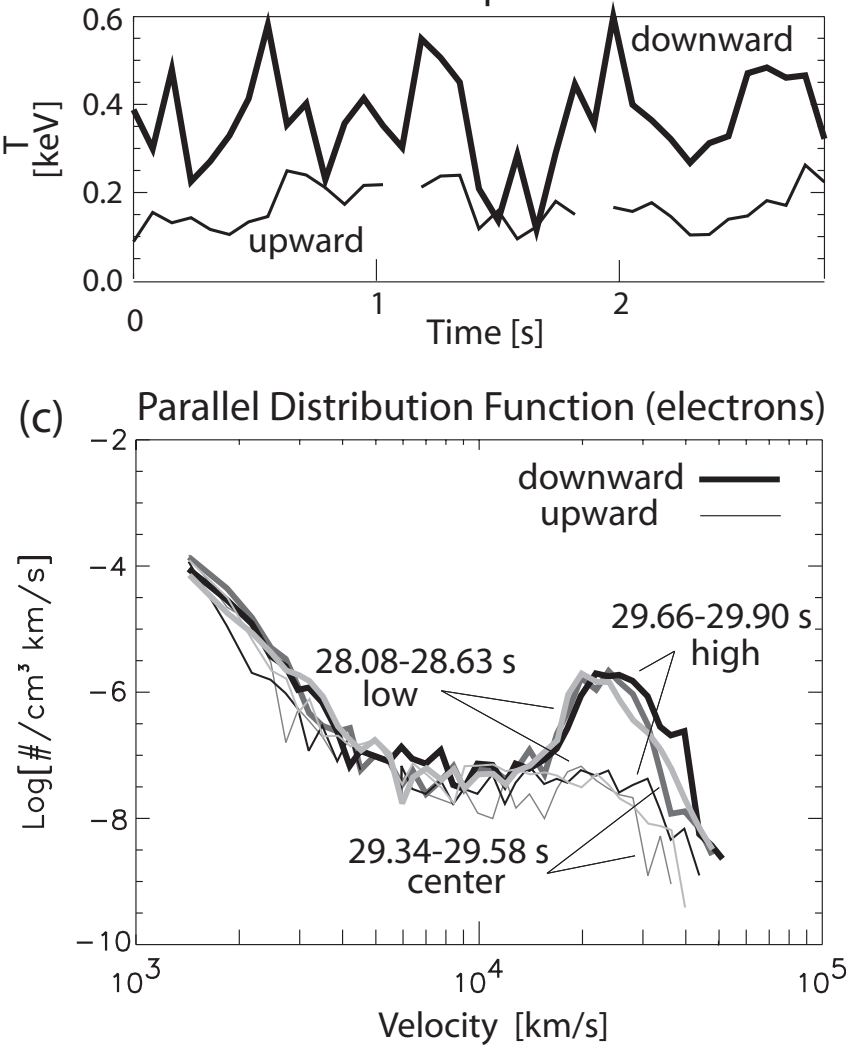

Fig. 2. (a) The ion and electron energy fluxes for the selected 3-s time interval exhibiting the anti-correlation in the energies of the upward ions and downward electrons (upper two panels). The lowest panel shows the upward electron energy flux being correlated with the downward much weaker electron flux. (b) Downward and upward electron temperatures. (c) Downward and upward parallel electron phase space densities (velocity distribution functions) in the three phases of electron energies: high, intermediate and low, corresponding to below, in the center and above the acceleration layer.

It has been demonstrated (Elphic et al., 1998;) that the upward current is indeed closed by the two downward currents such that a closed auroral current system is formed. Panel (b) shows the waveform (high-pass filtered above a few Hertz) which illustrates the strong electric activity in the upward current region (note that a direct measurement of the parallel component of a dc electric field present in the acceleration region is usually questionable), and panels (c) and (d) give an impression of the low and high frequency wave activity. In panel (d) the emission bands above frequency $f \sim 350 \mathrm{kHz}$ are events of auroral kilometric radiation, the strong radio signature of substorm activity. The horizontal line in this panel (d) is the electron cyclotron frequency $f_{c e}$. When the frequency of AKR is close to or below $f_{c e}$, this is conventionally taken as indication of the spacecraft being right in the radiation source region which is also the site of particle acceleration. The presence of several distinct emission bands indicates that several such acceleration zones may exist simultaneously at different altitudes.

Figure 1 suggests that spatially and temporarily the acceleration takes place at the two ends of the "inverted-V" region and proceeds in the very short time of 1-2s. Inside the "inverted-V" the parallel ion beam energy is fluctuating but its mean energy stays about constant, implying that the spacecraft is right in the acceleration zone and a substantial part of the electric acceleration potential is located below spacecraft orbit. Similar considerations apply to the parallel electron energy. However, closer inspection shows that the parallel energies of maximum ion and electron flux oscillate around their averages at a slow though irregular frequency.

Figure 2a shows the high resolution parallel ion and electron fluxes of the selected 3-s time interval measured in an opening angle of $\sim 20^{\circ}$. The upper two panels visualize a perfect anticorrelation of the maximum energy of the ion (upward) and electron (downward) energy fluxes. When the parallel upward energy of the ions increases, the parallel downward energy of the electrons decreases and vice versa, while the average energy remains constant. The variation in particle energy is irregular with quasi-periodicity of $\sim 1 \mathrm{~s}$. The bottom panel shows the upward electron fluxes measured during the same time interval. Their intensity exhibits exactly the same variation as the downward electron flux except that the energy of the upward electrons remains constant. This behavior is typical for ring distributions expected in the 
Electric Potential and Field Structure

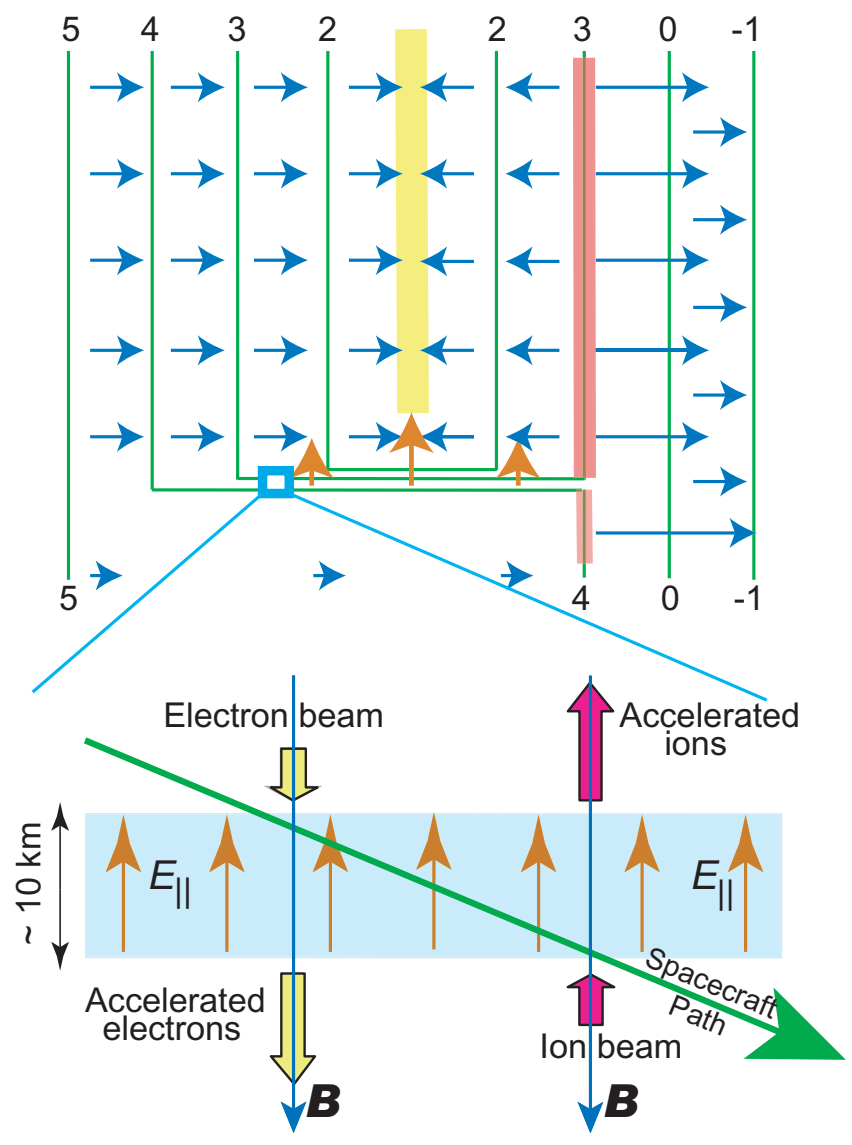

Fig. 3. Model of the electric potential structure, electric field (top) and the passage across the acceleration layer (bottom) which is only a small part (blue box) of the potential structure. Numbers on the lines indicate the hypothetical linearly increasing electric potential which mostly follows the magnetic lines of force. Blue arrows are perpendicular electric fields. The yellow bar is a hypothetical negative charge layer imposed by a converging convection. The red bar corresponds to diverging convection and a positive charge layer. Upward orange arrows indicate the upward electric field in the acceleration layer. The length of the arrows indicates the strength of the electric field. They increase towards the negative charge layer which is reflected by the slow increase in ion energy during the "inverted-V" event (Fig. 1). Note that these "charge layers" have not been inferred from the measurements as such measurements are impossible. They have been schematically introduced in order to complete the diverging or converging transverse electric fields and flows.

combination of acceleration in an electric potential field and scattering by the mirror force in a converging magnetic geometry.

In Fig. $2 b$ we plot the downward and upward electron temperatures. The downward electrons are about a factor of two hotter than the upward electrons while fluctuating strongly, depending on their phases, with highest temperatures in the intermediate phases between high and low electron energies. Finally, Fig. 2c shows the parallel phase space densities of upward and downward electrons in the high and low elec- tron energy phases as well as in the intermediate steep energy gradient phase (signified as "center"). The downward phase space distributions exhibit well expressed beams. The upward distribution in the high energy phase is plateaued out which is an indication of a nearly isotropic ring distribution and going on wave-particle interaction.

Quantitatively, the energization of the downward electrons and upward ions from their respective low to high energy levels is of the same order of magnitude. For instance, taking the transition in the ions in Fig. 2a at 1.2-1.8 s, the parallel increase in energy is $\sim 4 \mathrm{keV}$. This increase is matched by the successive increase in the parallel downward electron energy from 1.8-2.0 s, suggesting that the upward ions and downward electrons have successively crossed about the same potential difference of $\sim 4 \mathrm{kV}$. Since ions and electrons have been accelerated into opposite directions by this potential difference, and the ions are at their maximum energy level at the end of this crossing, the spacecraft must have passed a narrow layer of upward directed electric field from below the layer to above the layer and back to below the layer. Figure 3 shows the inferred electric potential and field structure and the scenario for the downward passage when the spacecraft traverses the layer from above to below. In this case the electrons are initially at low energy while the ions are at maximum energy. After passage the electrons are at high energy, and the ions are found to be at low energy. In the $0.6 \mathrm{~s}$ of the first (upward) crossing FAST has moved just $3 \mathrm{~km}$ in horizontal distance from south to north on this orbit. In the $0.2 \mathrm{~s}$ of the second (downward) crossing its horizontal displacement was merely $1 \mathrm{~km}$. Hence, the acceleration layer was either strongly folded in the vertical direction which would only be possible if it is very thin, or it moved up and down around the constant altitude of the spacecraft. Evidence for such motions had been obtained earlier from remote sensing observations of AKR (Pottelette et al., 2003) yielding estimated vertical (field-aligned) velocities the order of the ion acoustic speed (between $5-50 \mathrm{~km} / \mathrm{s}$ ) and an estimated width of $\sim 10 \mathrm{~km}$ for the accelerating layer. Panel (d) of Fig. 1 shows strong activity in AKR at frequencies below the local $f_{c e}$, indicating that FAST locally crosses the acceleration region, and a ring distribution in the electron phase space density has been formed. Adopting the higher velocity for the crossing, the width of the layer is $\sim 10 \mathrm{~km}$. A field aligned extension of this order is in agreement with a parallel speed of $\sim 20 \mathrm{~km} / \mathrm{s}$ in the slow crossing. This scenario is in accord with the appearance of upward electrons in the high electron energy phase which result from scattering in the combined magnetic and electric fields during the electron passage from above the layer to below the layer.

\section{Discussion and Conclusions}

From the former observations we may conclude that during the selected $3 \mathrm{~s}$ time sequence the spacecraft was midst in the auroral acceleration region where it is traversing an upward electric field acceleration layer. The relative position of the 

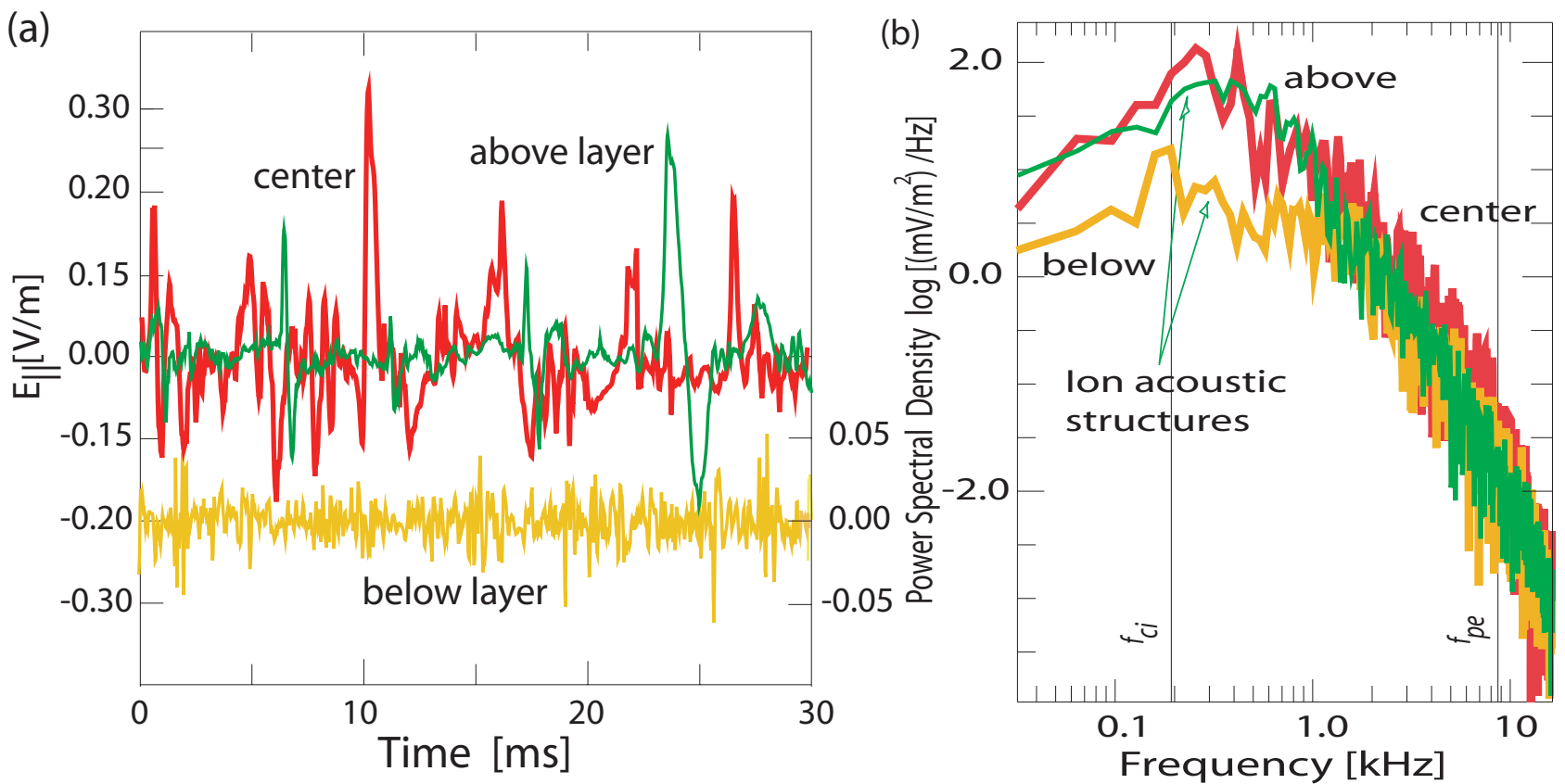

Fig. 4. Magnetic field-aligned electric wave forms (a) and power spectral densities (b) at three positions, above (green), in the center (red), and below (yellow) the acceleration layer for properly selected $30 \mathrm{~ms}$ time intervals. The parallel electric wave form above the center of the layer exhibit non-symmetric large-amplitude ion-acoustic solitary structures (ion holes) with net upward directed electric fields. In the center of the layer the electric turbulence is most intense with even stronger asymmetries parallel to the magnetic field but at the same time also much irregular than above the center, containing contributions from higher frequency. Small amplitude nearly unstructured high frequency (electron acoustic) turbulence is observed below the layer. The power spectral densities are high above the center of the layer, maximizing in the ion-acoustic range $(<600 \mathrm{~Hz})$, well above the ion-cyclotron frequency. Highest powers are seen in the center of the layer extending to high frequencies. Below the layer ion-cyclotron harmonics and weak high frequency electron acoustic waves are detected.

spacecraft with respect to this layer is fluctuating so that the spacecraft is sometimes above or below the acceleration region. The particle populations have previously been accelerated to an energy of about $1-2 \mathrm{keV}$ below the spacecraft in a layer which FAST had traversed at $8 \mathrm{~s}$ (see Fig. 1). A sketch of the scenario is given in Fig. 3. The question arises for the internal structure and the physics of this particular layer. In order to infer about their properties we take advantage of the availability of high-resolution measurements of the wave electric field parallel to the magnetic field. This is the direction where acceleration is going on.

Figure 4a shows $30 \mathrm{~ms}$ of the parallel electric wave form measured at three locations with respect to the acceleration layer, in the upper part of the layer (indicated as above layer) in the high ion energy/low electron energy phase, beneath the layer in the low ion energy/high electron energy phase, and in the center of the layer at the time of maximum gradient in ion energy. These data refer to the time interval 2.32-2.88 s during which one observes a parallel ion energy decrease (parallel electron energy increase) of $\sim 4 \mathrm{keV}$.

The electric field in all three positions is highly turbulent. There are, however, distinctions. The wave amplitudes are largest in the center of the acceleration layer in the average exceeding the fields above by at least a factor of 2 and below by an order of magnitude. Amplitudes reach more than $\sim 300 \mathrm{mV} / \mathrm{m}$ here. The character of the turbulence is rather ir- regular or chaotic with large non-symmetric structures dominating over a highly fluctuating background. It is hard to isolate single solitary structures here, but the positive excursions in the field clearly dominate. They correspond to upward directed fields in this case which are of the order of a few $100 \mathrm{mV} / \mathrm{m}$ and thus quite substantial.

Above the layer the low level of the field is quasiperiodically interrupted by large amplitude non-symmetric $\sim$ few ms period (in the ion acoustic range) bipolar electric field structures. The negative amplitudes obviously do not compensate for the positive ones yielding locally $(\sim 10-50 \mathrm{mV})$ net (upward) electric fields. These are presumably ion holes as detected two decades ago by Temerin et al. (1982) and at that time attributed to electrostatic ion cyclotron waves. These observations were later confirmed (Boström et al., 1988; Boström, 1992a, b; Ergun et al., 1998; Mozer and Hull, 2001) who rightly related them to ion acoustic modes. These holes are excited by the relative drifts of the different particle species (Hudson et al., 1983; Gray et al, 1991) including transverse velocity shear effects (Ganguli et al., 2002) which result in a significantly lower threshold current strength for the current-driven ion acoustic instability, and form so-called BGK modes (Muschietti et al., 1999; Umeda et al., 2002) of finite potential drop which in this case is upward the magnetic field. 
Below the layer the amplitudes of the turbulence are much less $(<50 \mathrm{mV} / \mathrm{m})$, and at low frequencies ion-cyclotron waves and harmonics appear.

The electric fluctuation spectra (Fig. 4b) are clearly dominated by those in the center of the layer where the maximum is in the ion-acoustic range above the ion cyclotron frequency, followed by power law decay of the spectrum, with power-law index $\xi \sim-2$, up to the plasma frequency where a cut-off is weakly indicated. The main power thus resides in the ion-acoustic modes but is modulated by the separation frequency of the single quasi-solitary structures. The plasma frequency derived from electrostatic analyzer data amounts to $f_{p e} \approx 8 \mathrm{kHz}$, and the cold $(<100 \mathrm{eV})$ electron population has density $n \sim 0.06 \mathrm{~cm}^{-3}$ yielding a cold plasma frequency of $f_{p c} \sim 2 \mathrm{kHz}$. Thus the high frequency turbulence is in accord with the reasonable assumption (Dubouloz et al., 1993) that electron acoustic turbulence contributes and is excited by the hot electrons in cooperation with the residual cold plasma.

The peak spectral power above the layer is above the ioncyclotron frequency $f_{c i}$, maximizing in the frequency range around $300 \mathrm{~Hz}$, typical for the presence of bi-polar ion holes excited in this region. In contrast, below the layer the dominant peak is around the ion-cyclotron frequency with indication of higher harmonics, which supports other observations (e.g. Chaston et al., 2002) of ion-cyclotron waves in relation to the auroral region. Comparison of these harmonics and the modulation of the spectrum in the center of the layer shows that the modulation is not due to the cyclotron harmonics but must be intrinsic to the generation of the quasisolitary ion hole structure in the center. At frequency above $600-800 \mathrm{~Hz}$ the spectrum merges into a steep power law with index $\xi \sim-3$, indicating the absence of high frequency turbulence above the center of the layer.

Finally, below the layer weak ion cyclotron harmonic activity is dominant (again in agreement with the findings of Chaston et al., 2002). A second peak in the spectrum is found in the range below $1 \mathrm{kHz}$ until at higher frequency the spectrum merges into a power law slightly flatter than that in the center and of only weakly reduced intensity. This higher frequency turbulence which maximizes around $f_{p c}$ can be identified with electron acoustic waves, possibly electron phase space holes generated by the interaction of the downward accelerated beam and cool upgoing electrons in the ring distribution (Dubouloz et al., 1993). Small scale electron holes produced cannot be resolved but move at faster than ion acoustic speed down with the electron beam along the field. The fastest of them leak out from the acceleration layer into the region below where in the spectrum they contribute to the high frequency peak.

The observe asymmetry in some of the solitary structures (holes) is of the order of $\sim 100 \mathrm{mV} / \mathrm{m}$ leading us to conclude that this is marginally enough to explain the observed acceleration of electrons and ions. The origin of the acceleration layer and the origin of the accelerating parallel electric field $E_{\|}$(e.g. Tetreault, 1991) is still under debate, however. Though the parallel electric fields taken from the soli- tary hole structures over the whole field aligned extension of the acceleration layer are large enough to explain at least part of the acceleration of the particles, it can not yet be proved unambiguously that their observation is the solution of the parallel auroral acceleration problem.

In this respect we comment on the question whether anomalous resistivity $\eta_{a n}=E_{\|} / j_{\|}$generated by instabilities of the field-aligned current $j_{\|}$in the upward current region, as suggested by several authors (e.g. Kindel and Kennel, 1971), could be responsible for the necessary potential drop $\Delta \Phi=-\int_{0}^{L} E_{\|} \mathrm{d} s$ along the magnetic field over the width $L$ of the acceleration layer. We can easily demonstrate that the anomalous resistivities required can hardly be produced by any of the known plasma instabilities. Indeed, the field-aligned current density can be obtained from panel (a) of Fig. 1 as $j_{\|}=\Delta B_{z} / \mu_{0} v_{s c} \Delta t$. With $\Delta B_{z} \approx 100 \mathrm{nT}$, $\Delta t=20 \mathrm{~s}$, and the spacecraft velocity of $v_{s c} \approx 5 \mathrm{~km} / \mathrm{s}$, the parallel current density is $j_{\|} \approx 8 \times 10^{-7} \mathrm{~A} / \mathrm{m}^{2}$, in agreement with the findings of Elphic et al.(1998). The acceleration potential amounts to $\Delta \Phi \approx(3-4) \mathrm{kV}$. Assuming $L=10 \mathrm{~km}$, this yields $\eta_{a n} \sim 4 \times 10^{5} / L_{10} \mathrm{Vm} / \mathrm{A}$, a rather high value for the collisionless plasma of the topside ionosphere at altitude of $3000 \mathrm{~km}$. The resistivity is defined through the anomalous collision frequency $v_{a n}$ as $\eta_{a n}=v_{a n} / \epsilon_{0} \omega_{p e}^{2}$, with $\omega_{p e}=2 \pi f_{p e}$. The observations yield $f_{p e}=8 \mathrm{kHz}$ corresponding to a density of the order of $n \sim 0.95 \mathrm{~cm}^{-3}$, which gives for the anomalous collision frequency

$v_{a n} \sim 0.01 n / L_{10} \approx 10^{4} / L_{10} \mathrm{~Hz}$.

Independent of any reasonable choice for the parallel extension of the acceleration layer long the magnetic field this value for the anomalous collision frequency is huge, of the order of the electron plasma frequency. No reasonable current or gradient driven plasma instability is known which could provide a collision frequency of this value. In fact, the theoretical ion acoustic collision frequency estimated for the above parameters is by at least four orders of magnitude below the required collision frequency. We may thus conclude that anomalous resistivities are incapable of generating the required potential drop and thus can be discarded as the agents of the observed particle acceleration in the aurora.

In summary, relatively narrow quasi-stationary turbulent electric acceleration layers seem to be responsible for part of the upward acceleration of auroral ions and downward acceleration of auroral electrons. When passing through the center of such layers one encounters an enhanced level of plasma turbulence which is characterized by an intense power spectral density in the ion-acoustic range, while the wave form is a mixture of large-amplitude structures and higher frequency turbulence. Above the center the turbulence is dominated by well expressed non-symmetric ion-acoustic like solitary structures exhibiting net upward directed electric fields and also carrying a substantial amount of the field that is necessary for acceleration. Below the layer the turbulence is weak of amplitude $<50 \mathrm{mV} / \mathrm{m}$. Altogether, the total potential drop across such a layer is of the order of $\mathrm{kV}$ (in the case of these observations) indicating that the layer comes up 
for a large fraction of the acceleration of electrons and ions. Hence a small number of such layers are required only to provide the total "inverted-V" electron energy in bright aurorae. It should be stressed that the observations discussed in the present paper are in good agreement with recent numerical simulation results on generation of holes (Singh et al., 1987; Singh and Khazanov, 2003). In particular, Singh et al. (1987) predicted the main features of panel (e) in Fig. 1 from simulations, namely a wide upward current sheet sandwiched between two return current regions. The self-consistent potential structure accelerating the upward and downward accelerated electrons and ions was found from 2-D PIC simulations of current sheet equilibrium by varying the width of the upward current sheet. In addition, they predicted the different types of turbulence above and in the center of the acceleration layer (their Figs. 10 and 13, the contours H, there, show the spiky fields of transient double layers, which contribute to the downward accelerations of electrons and upward accelerations of ions).

We note once more that the mechanism of formation of such layers remains essentially unclarified. We believe that the mechanism of formation is closely related to the presence of shear flows, as indicated in Fig. 3. Note that in the present cases shear motions (converging perpendicular electric fields) are highly reasonable to exist in the entire field-aligned potential drop (acceleration) zone as they are required by the potential structure. Ganguli (1997) has shown that the effect of such velocity shear on the lowfrequency kinetic Kelvin-Helmholtz instability is very important. Though the Kelvin-Helmholtz instability in the kinetic regime is strongly Landau damped, a new branch of shear velocity driven instability arises which produces broadband electric turbulence in the perpendicular direction, with frequency ranging from the ion cyclotron frequency $f \sim f_{c i}$ up to $\sim 10 f_{c i}$. This instability is not sensitive to the details of the shear flow. Figure 4 refers to parallel turbulence, however, and thus does not contain contributions of this kind of oscillations.

In conclusion we note that, thanks to the high time resolution measurements performed on board the FAST spacecraft including both wave and particle distributions, it has been possible to clearly identified the characteristics of the turbulence generated inside a shock structure located in the upward current region. At each level, nonlinear electron and ion acoustic structures appear to play a key role in sustaining the shock and producing localized small-scale net upward electric fields.

Acknowledgements. The authors thank M. Berthomier, R. Ergun, J. McFadden, and C. Carlson for discussions.

Edited by: G. S. Lakhina

Reviewed by: G. I. Ganguli and another referee

\section{References}

Block, L. P. and Fälthammar, C.-G.: The role of magnetic fieldaligned electric fields in auroral acceleration, J. Geophys. Res., 95, 5877-5888, 1990.

Boström, R.: Voltage drops along auroral magnetic field lines on small and large scale, Phys. Space Plasmas, 15, 37-49, 1992a.

Boström, R.: Observations of weak double-layers on auroral field lines, IEEE transactions on plasma science 20, 756-763, $1992 \mathrm{~b}$.

Boström, R., Gustafsson, G., Holback, B., Holmgren, G., and Koskinen, H.: Characteristics of solitary waves and weak double layers in the magnetospheric plasma, Phys. Rev. Lett., 61, 82-85, 1988.

Carlson, C. W., Pfaff, R. F., and Watzin, J. G.: The Fast Auroral Snapshot (FAST) mission, Geophys. Res. Lett., 25, 2013-2016, 1998a.

Carlson, C. W., McFadden, J. P., Ergun, R. E., et al. : FAST observations in the downward auroral current region: Energetic upgoing electron beams, parallel potential drops, and ion heating, Geophys. Res. Lett., 25, 2017-2020, 1998b.

Chaston, C. C., Bonnell, J. W., McFadden, J. P., Ergun, R. E., and Carlson, C. W.: Electromagnetic ion cyclotron waves at proton cyclotron harmonics, J. Geophys. Res., 107, 1-19, 2002.

Chiu, Y. T. and Schulz, M.: Self-consistent particle and parallel electrostatic field distributions in the magnetosphericionospheric auroral region, J. Geophys. Res. 83, 629-642, 1978.

Dubouloz, N., Treumann, R. A., and Pottelette, R.: Turbulence generated by a gas of electron-acoustic solitons, J. Geophys. Res., 98, 17415-17 422, 1993.

Elphic, R. J., Bonnell, J. W., Strangeway, R. J., et al.: The auroral current circuit and field-aligned currents observed by FAST, Geophys. Res. Lett., 25, 2033-2036, 1998.

Ergun, R. E., Carlson, C. W., McFadden, J. P., et al.: FAST satellite observations of large amplitude solitary structures, Geophys. Res. Lett., 25, 2041-2044, 1998.

Ganguli, G.: Stability of an inhomogeneous transverse plasma flow, Phys. Plasmas, 4, 1544-1551, 1997.

Ganguli, G., Shinkler, S., Gavrishchaka, V., and Scales, W.: Low frequency oscillations in a plasma with spatially variable fieldaligned flow, Phys. Plasmas, 9, 2321, 2002.

Gray, P., Hudson, M. K., Lotko, W., and Bergmann, R.: Decay of ion beam driven acoustic waves into ion holes, Geophys. Res. Lett., 18, 1675-1678, 1991.

Gurnett, D. A. and Anderson, R. R.: The kilometric radioemission spectrum: relationship to auroral acceleration processes, in Physics of auroral arc formation, edited by Akasofu, S.-I. and Kan, J. R., AGU, Washington, D.C., Geophys. Monogr. 25, 341350, 1981.

Hudson, M. K., Lotko, W., Roth, I., and Witt, E.: Solitary waves and double layers on auroral field lines, J. Geophys. Res., 88, 916-926, 1983.

Marklund, G., Karlsson, T., and Clemmons, J.: On low-altitude particle acceleration and intense electric fields and their relation to black aurora, J. Geophys. Res., 102, 17 509-17 522, 1997.

Marklund, G. T., Ivchenko, N., Karlsson, T., et al: Temporal evolution of the electric field accelerating electrons away from the auroral ionosphere, Nature, 414, 724-727, 2001.

McFadden, J. P., Carlson, C. W., Ergun, R. E., Mozer, F. S., Muschietti, L., Roth, I., and Moebius, E.: FAST observations of ion solitary waves, J. Geophys. Res., 108, 8018, COA 19-1-19-7, doi:10.1029/2002JA009485, 2003. 
Mozer, F. S. and Hull, A.: Origin and geometry of upward parallel electric fields in the auroral acceleration region, J. Geophys. Res., 106, 5763-5778, 2001.

Muschietti, L., Ergun, R. E., Roth, I., and Carlson, C. W.: Phasespace electron holes along magnetic field lines, Geophys. Res. Lett., 26, 1093-1096, 1999.

Pfaff, R., Carlson, C., Watzin, J., et al.: An overview of the Fast Auroral SnapshotT (FAST) satellite, Space Sci. Rev., 98, 1-32, 2001.

Pottelette, R., Treumann, R. A., and Berthomier, M.: Auroral plasma turbulence and the cause of auroral kilometric radiation fine structure, J. Geophys. Res., 106, 8465-8476, 2001.

Pottelette, R., Treumann, R. A., Berthomier, M., and Jasperse, J.: Electrostatic shock properties inferred from AKR fine structure, Nonlin. Proc. Geophys. 10, 87-92, 2003.
Singh, N., Thiemann, H., and Schunk, R. W.: Simulations of auroral plasma processes: Electric fields, waves and particles, Planet. Space Sci., 35, 353-395, 1987.

Singh, N. and Khazanov, G.: Double layers in expanding plasmas and their relevance to the auroral plasma processes, J. Geophys. Res., 108, 8007, COA 8-1-8-16, 2003.

Temerin, M., Cerny, K., Lotko, W., and Mozer, F. S.: Observations of double layers and solitary waves in the auroral plasma, Phys. Rev. Lett., 48, 1175-1179, 1982.

Tetreault, D.: Theory of electric fields in the auroral acceleration region, J. Geophys. Res., 96, 3549-3561, 1991.

Umeda, T., Omura, Y., Matsumoto, H., and Usui, H.: Formation of electrostatic solitary waves in space plasmas: Particle simulations with open boundary conditions, J. Geophys. Res., 107, SMP 19-1-19-16, 1449, doi:10.1029/2001JA000286, 2002. 